\title{
Análisis de las relaciones emociones-aprendizaje de maestros en formación inicial con una práctica activa de Biología
}

\author{
José María Marcos-Merino \\ Departamento de Didáctica de las Ciencias Experimentales y las Matemáticas, Facultad de Educación, \\ Universidad de Extremadura.Badajoz,España.jmmarcos@unex.es \\ ORCID: bttp:// orcid.org/0000-0003-0773-2899
}

[Recibido: 1 Octubre 2018. Revisado: 4 Octubre 2018. Aceptado: 12 Noviembre 2018]

Resumen: El alumnado experimenta una gran diversidad de emociones en los distintos contextos académicos. Estas emociones y el aprendizaje de las ciencias se condicionan recíprocamente, por lo que es necesario abordar simultáneamente los aspectos afectivos y cognitivos en los procesos de enseñanza. Esto es particularmente importante en los maestros en formación, ya que la relación entre sus emociones y su aprendizaje puede determinar su futuro desempeño profesional. Para profundizar en estas interacciones, en el presente trabajo se analizan las emociones experimentadas por una muestra de estudiantes del Grado de Maestro en Educación Primaria (Universidad de Extremadura), antes y después de la implementación de una práctica activa de Biología; y su nivel de conocimientos de Biología, tanto previos como adquiridos con la intervención. Los resultados revelan asociaciones positivas entre la intensidad de las emociones alegría, entusiasmo, satisfacción y diversión experimentadas durante la práctica y los resultados de aprendizaje, al igual que asociaciones negativas entre estos y la intensidad de la frustración y la preocupación. Asimismo, los resultados indican que bajos niveles de conocimientos previos de Biología son predictores de altas intensidades de aburrimiento, frustración y preocupación, así como que la intensidad previa de entusiasmo puede tener valor predictivo en relación al aprendizaje.

Palabras clave: Emociones, aprendizaje, formación inicial de maestros, valor predictivo.

Relationships between emotions and learning outcomes in pre-service Primary teachers towards an active Biology practice

\begin{abstract}
Students experience a great diversity of emotions in academic settings. These emotions and science learning are reciprocally conditioned, therefore it is important to simultaneously look into affective and cognitive aspects in learning processes. This is particularly important in prospective Primary teachers, since the interplay between emotions and learning can determine its future professional. To deepen these interactions, in this contribution we analyse the relationships between the emotions experienced by a sample of students of the Degree in Primary Education (University of Extremadura), before and after the implementation of an active practice of Biology; and their level of Biology knowledge, both previous and acquired with the performed intervention. Results reveal positive associations between the intensity of joy, enthusiasm, satisfaction and fun experienced during the practice and learning outcomes, as well as negative associations between them and the intensities of frustration and worry. Likewise, results indicate that low levels of previous Biology knowledge are predictors of high intensities of boredom, frustration and worry, as well as the previous intensity of enthusiasm can have predictive value in relation to learning.
\end{abstract}

Keywords: Emotions, learning, initial teacher training, predictive value.

Para citar este artículo: Marcos-Merino, J.M. (2019) Análisis de las relaciones emociones-aprendizaje de maestros en formación inicial con una práctica activa de Biología. Revista Eureka sobre Enseñanza y Divulgación de las Ciencias 16(1), 1603. doi: 10.25267/Rev_Eureka_ensen_divulg_cienc.2019.v16.11.1603

\section{Introducción}

Las aulas son ambientes emocionales: en el ámbito académico se experimentan las principales emociones humanas. No obstante, y a pesar de su influencia en el aprendizaje, la Didáctica de las Ciencias se ha centrado más en el dominio cognitivo que en el afectivo, al considerar a las

\author{
Revista Eureka sobre Enseñanza y Divulgación de las Ciencias \\ Universidad de Cádir. APAC-Eureka. ISSN: 1697-011X \\ bttp:/ / dx.doi.org/10.25267/Rev_Eureka_ensen_divulg_cienc.2019.v16.i1.1603 \\ http:/ / reuredc.uca.es
}


emociones como elementos acientíficos que se oponían a la objetividad de la Ciencia (Mellado et al. 2014). Hoy día, sin embargo, se considera que las emociones son un producto evolutivo con el que valorar los eventos que afronta el ser humano y estimar si son deseables para su futuro (Damasio 2010). Así, para Bisquerra (2000) las emociones son reacciones a la información recibida del entorno, cuya intensidad depende de las evaluaciones subjetivas que el individuo realiza. En definitiva, una emoción depende de lo que es importante para el individuo. Damasio (2010) añade que las emociones no sólo son reacciones a los estímulos del presente, sino que también se producen por el recuerdo o evocación de hechos sucedidos en el pasado o por el anticipo de posibles situaciones futuras.

\section{Las emociones y su relación bidireccional con el aprendizaje}

Las emociones influyen en el aprendizaje, ya que regulan los niveles de atención, intervienen sobre la motivación para aprender y condicionan la elección de las estrategias de aprendizaje (Bradley 2000; Mega, Ronconi y De Beni 2014). Asimismo, se ha comprobado su influencia en la memoria: la información relacionada con algún estímulo emocional se recuerda mejor que la neutra (Dunsmoor, Murty, Davachi y Phelps 2015). De este modo, se ha constatado que una activación de las emociones positivas puede dar lugar a mejores resultados de aprendizaje; ya que los estímulos emocionales positivos provocan, a nivel cerebral, una excitación que mejora la memoria (Fredrickson 1998; Kensinger y Corkin 2004) y los niveles de atención y de reflexión (Aydogan, Bozkurt y Coskun 2015). Estas observaciones se apoyan en diferentes estudios neurofisiológicos que muestran como, en el cerebro, las emociones y los procesos cognitivos (la memoria, la atención o la capacidad de resolver problemas) están integrados en áreas críticas para la regulación del flujo de información entre regiones (Gu, Liu, Van Dam, Hof y Fan 2013). La neurociencia indica que las emociones "encienden" las conexiones para que el cerebro alcance el máximo de sus funciones cognitivas (Mora 2008). Así, se ha demostrado que frecuentemente las emociones positivas se asocian positivamente con el aprendizaje, mientras que este se asocia negativamente con las emociones negativas (Pekrun, Goetz, Frenzel, Barchfeld y Perry 2011).

Estudios neurofisiológicos han mostrado que los dominios afectivo y cognitivo están recíprocamente condicionados: las emociones influyen en el aprendizaje a la vez que los resultados de aprendizaje en las emociones (Eldar y Niv 2015). En un contexto académico las emociones están implicadas en la estimación previa que realiza el alumno para valorar el esfuerzo a realizar en función de las futuras recompensas, internas (como la superación personal) o externas (por ejemplo sus calificaciones). La precisión con la que se ha realizado esta estimación, una vez obtenidas o no las recompensas, modifica a su vez las emociones del alumno (Eldar, Rutledge, Dolan y Niv 2016). Esto refuerza el papel evolutivo de las emociones y su importancia en el aula (Damasio 2010), ya que el aprendizaje es una tarea cuyo resultado es incierto para los alumnos. En base a estas interacciones se ha propuesto que las emociones ante una actividad pueden tener valor predictivo de los resultados de aprendizaje a obtener con la misma. De este modo, se ha demostrado que una alta intensidad previa de emociones positivas predice buenos resultados de aprendizaje mientras que una alta intensidad de emociones negativas predice malos resultados (Pekrun, Goetz, Daniels, Stupnisky y Perry 2010; Pekrun, Goetz, Titz y Perry 2002; Ruthig et al. 2008). Dadas las relaciones entre emociones y aprendizaje, la influencia entre ambas es también bidireccional. Así, se ha corroborado que los resultados de aprendizaje del pasado influyen en las emociones hacia actividades del presente: los buenos resultados como predictores de emociones positivas y los malos resultados de emociones negativas (Pekrun et al. 2002; Pekrun, Elliot y Maier 2006; Pekrun, Elliot y Maier 2009). 


\section{Las emociones en la enseñanza de las ciencias: implicaciones en la formación inicial de maestros}

Teniendo en cuenta la relación entre las emociones y el aprendizaje, se ha recomendado que los docentes consideren los aspectos afectivos en los procesos de enseñanza (Mellado et al. 2014). Además, se ha demostrado que las emociones experimentadas por los maestros hacia los contenidos científicos, y hacia su enseñanza, se transfieren a sus alumnos (Frenzel, Goetz, Lüdtke, Pekrun y Sutton 2009). Por ello, actualmente la investigación aconseja que se utilicen las emociones positivas que experimentan los profesores para promover estas entre sus alumnos (Damasio 2010), estableciendo la necesidad de generar emociones positivas hacia la enseñanza de las ciencias desde la formación inicial del profesorado. Esto resulta relevante en relación a los maestros en formación inicial, ya que se ha mostrado que su recuerdo de emociones hacia las ciencias en Educación Secundaria (etapa en la que decae la intensidad de sus emociones positivas al tiempo que aumenta la frecuencia de las negativas) se transfiere a las que creen que van a sentir cuando impartan clases de estos contenidos. Además, dicho recuerdo se corresponde con lo que realmente sienten cuando imparten ciencias durante sus prácticas de enseñanza (Brígido, Borrachero, Bermejo y Mellado 2013).

Una manera de generar emociones positivas es la implementación de actividades prácticas. Sin embargo, los resultados de las prácticas tradicionales, basadas en seguir las instrucciones cerradas establecidas en un protocolo, no son satisfactorios (Reigosa y Jiménez 2000). Por ello, desde el constructivismo, se ha propuesto dotar a las prácticas de un enfoque investigativo; planteando situaciones abiertas que exigen la búsqueda de respuestas por parte de los alumnos, mediante pequeñas investigaciones desarrolladas bajo la supervisión del profesor. Numerosos estudios han comprobado que estas actividades basadas en la investigación dirigida generan buenos resultados de aprendizaje (Gormally, Brickman, Hallar y Amstrong 2009); al tiempo que provocan, respecto a la expectativa inicial de los alumnos, una mayor frecuencia de emociones positivas y una disminución en la frecuencia de las negativas (RetanaAlvarado, De las Heras-Pérez, Vázquez-Bernal y Jiménez-Pérez 2018).

Sin embargo, a pesar de su importancia en la Enseñanza de las Ciencias, los profesores de Educación Primaria en ejercicio reconocen que realizan pocas prácticas en la enseñanza de las ciencias (García-Barros y Martínez-Losada 2001). Dada esta circunstancia, es necesario incluir, en los programas de formación inicial de docentes, actividades prácticas activas que fomenten las emociones positivas hacia los contenidos científicos y hacia su enseñanza-aprendizaje (Damasio 2010). Sin embargo, es necesario realizar estudios que midan la influencia de estas actividades en las emociones de los alumnos y en el aprendizaje de las ciencias; así como profundizar en el estudio de las interacciones entre ambos fenómenos. Actualmente, desde nuestro grupo de investigación, estamos indagando en el papel de las emociones en el aprendizaje de ciencias de maestros en formación inicial mediante prácticas activas. Para ello hemos diseñado y validado, mediante análisis factorial, un sencillo y rápido test cuantitativo autoinforme con el que estimar la intensidad de una serie de emociones académicas (MarcosMerino, Esteban y Gómez 2016). Resultados preliminares muestran que una práctica activa de Biología, desarrollada bajo indagación guiada, mejora las emociones de los alumnos respecto a su expectativa inicial (aumento de la intensidad de las emociones positivas y disminución de la intensidad de las negativas); así como que es efectiva en relación al aprendizaje (MarcosMerino et al. 2016). Además, este estudio, en el que las emociones mostraron un comportamiento factorial que permitía calcular un valor global para el conjunto de las emociones positivas o negativas; ha revelado una asociación positiva entre el factor correspondiente a las emociones positivas sentidas durante la práctica y los resultados de aprendizaje. Sin embargo, no se ha profundizado en el análisis de las relaciones establecidas 
entre la intensidad de cada emoción (antes y después de la implementación de dicha práctica) y el nivel de conocimientos de Biología (tanto previos como adquiridos con la intervención).

\section{Objetivos}

El objetivo principal planteado en el presente trabajo es determinar si existen asociaciones significativas entre las emociones experimentadas antes y después de la implementación de una práctica activa de Biología, el nivel de conocimientos previos y los resultados de aprendizaje alcanzados con dicha intervención práctica, en una muestra de estudiantes del Grado de Maestro en Educación Primaria de la Universidad de Extremadura. Para ello se plantean varios objetivos específicos:

- Analizar las asociaciones entre el nivel de conocimientos previos de Biología de Educación Secundaria de los participantes y la intensidad de emociones esperada ante una intervención práctica incluida en su programa de formación como maestros.

- Analizar las asociaciones entre la intensidad de emociones experimentadas por los participantes durante una práctica activa de Biología y los resultados de aprendizaje obtenidos con esta.

- Analizar las asociaciones entre los resultados de aprendizaje alcanzados por los participantes y su expectativa de emociones antes de la práctica, a fin de determinar si la expectativa de emociones ante la actividad tiene un valor predictivo en los resultados de aprendizaje.

\section{Metodología}

\section{Muestra}

La muestra está formada por 159 maestros en formación inicial (21.8 años de media; $66.33 \%$ de mujeres), estudiantes del Grado de Maestro en Educación Primaria de la Universidad de Extremadura. Dichos participantes pertenecen a tres grupos de estudiantes que cursaban la asignatura Didáctica del Medio Físico y los Seres Vivos, del tercer curso de dicho grado. Respecto a sus estudios en Educación Secundaria, sólo un 31 \% de los participantes cursó una modalidad de ciencias en Bachillerato.

\section{Instrumento empleado para estimar las emociones}

Para estimar las emociones de los participantes se emplea un test autoinforme cuantitativo formado por ítems sencillos. El muestreo mediante cuestionarios en los que los participantes autoinforman de sus emociones es el procedimiento más utilizado actualmente para estudiar las emociones de los alumnos, ya que constituye una medida rápida que apenas afecta al desarrollo de las actividades del aula (Mauss y Robinson 2009). Además, dicho test emplea ítems simples, que comparados con test multi-ítem, más largos, proporcionan suficiente validez, requieren menos tiempo y son menos invasivos en las respuestas emocionales de los participantes. El cuestionario incluye 10 emociones: 5 positivas (alegría, confianza, satisfacción, entusiasmo y diversión) y 5 negativas (preocupación, frustración, incertidumbre, nerviosismo y aburrimiento), seleccionadas de entre todas las emociones que los alumnos pueden experimentar durante los procesos de enseñanza-aprendizaje (Mellado et al. 2014). Los alumnos autoinforman, siguiendo una escala ordinal de Likert (que oscila desde 1 "no experimentada" a 5 "intensamente experimentada"), sobre la intensidad con la que creían que 
iban a experimentar (antes de la intervención) o habían experimentado (15 días después de la implementación de la intervención) dichas emociones. Así, el test diseñado interroga sobre una de las dimensiones utilizadas para estudiar las emociones: la valencia (diferenciación entre emociones positivas y emociones negativas), a la vez que estudia la intensidad con que se experimenta cada emoción.

Este test autoinforme de emociones ha sido validado en un trabajo previo con una muestra de maestros en formación inicial. Dicha validación se basa en su comportamiento factorial (Marcos-Merino et al. 2016) y en la concordancia de este con numerosos estudios previos en los que se han desarrollado cuestionarios para estimar emociones (Pekrun et al. 2011). Ahondando en su comportamiento factorial, el estudio de intercorrelaciones entre la intensidad de las emociones analizadas en distintos contextos académicos, presentes y pasados, mostró asociaciones positivas entre emociones que tienen la misma valencia (positiva o negativa); así como una falta de asociación entre emociones de valencia opuesta. Los resultados del análisis factorial respaldaron estas observaciones, confirmando la existencia de factores latentes comunes al conjunto de las emociones positivas o negativas en cada uno de los contextos analizados.

\section{Instrumento de evaluación de los conocimientos en Biología}

Para evaluar el grado de conocimiento de conceptos básicos de Biología Celular y Genética (áreas biológicas relacionadas con la intervención implementada) se emplea un cuestionario (anexo 1) formado por 14 preguntas cerradas de tipo test (con una única opción correcta). Dicho cuestionario se implementa antes de la intervención (para determinar los conocimientos previos de los participantes) y 15 días después de su realización (para establecer los resultados de aprendizaje alcanzados). El procedimiento de elaboración y validación de este instrumento sigue los pasos indicados por Smith, Wood y Knight (2008):

- En primer lugar, se realizó una revisión bibliográfica sobre concepciones alternativas relacionadas con ambas áreas biológicas en Educación Secundaria Obligatoria (ESO) y en maestros en formación inicial (Banet y Ayuso 2003; Caballero 2008; Chattopadhyay 2005; González-Weil y Harms 2012; Marcos-Merino y Esteban 2018) La razón por la cual se emplean concepciones alternativas comunes entre alumnos de ESO es que esta etapa educativa es la última en la que la mayoría de los participantes cursaron asignaturas de ciencias.

- En segundo lugar, dada esta circunstancia, se seleccionaron los objetivos de aprendizaje de ESO que están relacionados con ambas disciplinas biológicas. En base a ellos, se elaboraron algunas preguntas, a la vez que se extrajeron preguntas relacionadas del TIMSS para $8^{\circ}$ grado, $2^{\circ}$ de ESO en el caso de España (Foy, Arora y Stanco 2013).

\section{Procedimiento}

Los estudiantes cumplimentaron los instrumentos anteriormente descritos antes y 15 días después de realizar un seminario práctico sobre Biología Celular, Genética y su didáctica en Primaria. Antes de ello, fueron informados de los objetivos de la investigación, y del carácter voluntario y anónimo de su participación. El tiempo medio empleado por los participantes para rellenarlos osciló entre 10 y 15 minutos. En dicho seminario se realizó una práctica de extracción de DNA, de tomate y de células de la mucosa bucal, con materiales cotidianos (sal, bicarbonato sódico, detergente lavavajillas y alcohol). La metodología seguida en esta práctica se ha descrito pormenorizadamente en un trabajo previo (Marcos-Merino et al. 2016), y se basa en la indagación guiada. Se considera una práctica activa ya que, en ella, el docente plantea diversos problemas en forma de preguntas abiertas, que los estudiantes deben 
responder mediante debates, en los que utilizan sus conocimientos previos, y realizan pequeñas investigaciones bajo la dirección del profesor. El alumno tiene un papel activo en la resolución de los problemas planteados, así como en la elaboración y ejecución de su propio protocolo de extracción. El análisis de los resultados de ambos cuestionarios ha mostrado que la implementación de este seminario disminuyó la frecuencia de emociones negativas de los participantes, así como aumentó sus emociones positivas y sus conocimientos de Biología (Marcos-Merino et al. 2016).

\section{Análisis estadístico}

Dado que los datos no se ajustaban a una distribución normal se utilizó estadística no paramétrica. Los datos de los tres grupos de estudiantes se han agrupado en un único conjunto de datos, tras comprobar la ausencia de diferencias significativas entre ellos. Los test de normalidad (Kolmogoro-Smirnov y Shapiro-Wilk) y los análisis de correlación (Spearman) se realizaron con el programa SPSS v25 (IBM software). Para realizar correlaciones se emplean valores centrados de las notas y las emociones (calculados restando la media y dividiendo por la desviación estándar).

\section{Resultados y discusión}

Análisis de las relaciones entre la expectativa de emociones ante una práctica de Biología del presente y el recuerdo de conceptos de Biología de Educación Secundaria

Los estudios de correlación de Spearman reflejan que existe una asociación significativa entre la intensidad de algunas emociones negativas, que los participantes esperan experimentar con una práctica de Biología incluida en su programa formativo; y su grado de recuerdo de conceptos biológicos básicos impartidos en Educación Secundaria (medidos a través de la nota del pretest) (Tabla 1). De este modo, se han detectado correlaciones negativas entre la intensidad esperada de aburrimiento ( $\mathrm{p}$-valor=.043), frustración ( $\mathrm{p}$-valor=.003) y preocupación ( $\mathrm{p}$-valor=.015), y el nivel de conocimientos previos de Biología de Educación Secundaria.

Tabla 1. Coeficientes de correlación de la intensidad de las emociones previas a una intervención práctica con el grado de recuerdo de conceptos de Biología de Educación Secundaria (nota del pretest). En negrita se resaltan las correlaciones significativas (Correlación de Spearman, ${ }^{* *}$ p-valor $<.01,{ }^{*}$ p-valor $\left.<.05\right)$

\begin{tabular}{|c|c|}
\hline $\begin{array}{c}\text { Expectativa de emociones ante una } \\
\text { práctica de Biología }\end{array}$ & $\begin{array}{c}\text { Coeficiente de correlación con el recuerdo de } \\
\text { conceptos de Biología de Educación Secundaria }\end{array}$ \\
\hline Alegría & .069 \\
\hline Confianza & .079 \\
\hline Satisfacción & .071 \\
\hline Entusiasmo & .125 \\
\hline Diversión & .121 \\
\hline Nerviosismo & -.062 \\
\hline Aburrimiento & $-.157^{*}$ \\
\hline Frustración & $-.227^{* *}$ \\
\hline Preocupación & $-.190^{*}$ \\
\hline Incertidumbre & -.011 \\
\hline
\end{tabular}

Esta observación indica que aquellos alumnos que esperan sentir más aburrimiento, frustración y preocupación durante una práctica de Biología del Grado de Maestro en Educación Primaria, son aquellos que recuerdan en menor grado los contenidos de Biología impartidos en Educación Secundaria. Estos resultados están en consonancia con diversos estudios previos que han comprobado la influencia de los resultados de aprendizaje del pasado 
en las emociones de los alumnos hacia actividades del presente (Pekrun et al. 2002; Pekrun et al. 2006; Pekrun et al. 2009). En concreto, las correlaciones detectadas concuerdan con la observación de los malos resultados de aprendizaje como predictores de emociones negativas, relación detectada en estas investigaciones previas para el aburrimiento, la ira, la vergüenza y la desesperación. Estos estudios también encontraron relaciones significativas entre los resultados de aprendizaje del pasado y las emociones positivas del presente (en concreto los buenos resultados como predictores de entusiasmo, ilusión y orgullo), correlaciones no detectadas en la presente investigación. Se requieren, por tanto, futuros estudios con los que profundizar en la influencia del nivel de conocimientos previos de ESO de los futuros maestros en sus emociones, cuya conexión con las emociones que experimentaron como alumnos de esta etapa educativa ha sido descrita por Brígido et al. (2013).

\section{Análisis de las relaciones entre las emociones experimentadas con una práctica activa de Biología y los resultados de aprendizaje}

Los análisis de correlación reflejan una asociación significativa entre la intensidad de algunas emociones experimentadas durante la práctica activa implementada, sobre todo positivas, y los resultados de aprendizaje alcanzados con esta, medidos mediante la nota del postest (Tabla 2). Así, se observan correlaciones positivas entre la nota del postest y la intensidad de alegría ( $\mathrm{p}$ valor $<.001$ ), satisfacción ( $\mathrm{p}$-valor $<.001)$, entusiasmo ( $\mathrm{p}$-valor $<.001)$ y diversión ( $\mathrm{p}$-valor $=.007$ ) sentidas durante la implementación de la intervención. Por otro lado, se aprecian correlaciones negativas entre la nota del postest y las intensidades de frustración ( $\mathrm{p}$-valor=.011) y preocupación (p-valor=.013). Estos resultados sugieren que los alumnos que obtuvieron mejores resultados de aprendizaje tras la implementación de la práctica activa de Biología, son aquellos que sintieron más alegría, satisfacción, entusiasmo y diversión; así como menos frustración y preocupación, durante el desarrollo de la misma.

Tabla 2. Coeficientes de correlación de la intensidad de las emociones sentidas durante la práctica activa con los resultados de aprendizaje alcanzados tras su implementación (nota del postest). En negrita se resaltan las correlaciones significativas (Correlación de Spearman, *** pvalor $<.001, * * \mathrm{p}$-valor $<.01, *$ p-valor $<.05$ )

\begin{tabular}{|c|c|}
\hline $\begin{array}{c}\text { Emociones experimentadas con } \\
\text { una práctica activa de Biología }\end{array}$ & $\begin{array}{c}\text { Coeficiente de correlación con los resultados de } \\
\text { aprendizaje }\end{array}$ \\
\hline Alegría & $.276^{* * *}$ \\
\hline Confianza & .130 \\
\hline Satisfacción & $.278^{* * *}$ \\
\hline Entusiasmo & $.279^{* * *}$ \\
\hline Diversión & $.213^{* *}$ \\
\hline Nerviosismo & -.056 \\
\hline Aburrimiento & -.149 \\
\hline Frustración & $-.200^{*}$ \\
\hline Preocupación & $-.196^{*}$ \\
\hline Incertidumbre & -.068 \\
\hline
\end{tabular}

Estos resultados coinciden con investigaciones previas que han hallado asociaciones significativas entre los resultados de aprendizaje y las emociones experimentadas por los alumnos en el aula durante su adquisición (Marcos-Merino, Esteban y Gómez 2019; Pekrun et al. 2011): las emociones positivas se asocian positivamente con el aprendizaje mientras que las emociones negativas lo hacen negativamente. También concuerdan con un estudio previo, en el que se analizaron las emociones hacia esta práctica activa de Biología como valores globales mediante factores, que detectó una asociación positiva entre el factor asociado a las emociones positivas sentidas durante dicha práctica y los resultados de aprendizaje (Marcos-Merino et al. 2016). Sin embargo, dicho trabajo no detectó asociaciones con el factor asociado a las 
emociones negativas. A pesar de ello, se observa que algunas emociones a nivel individual (en concreto la frustración y la preocupación sentidas durante la práctica) sí se asocian con los resultados de aprendizaje. Además, a pesar de la correlación detectada previamente entre el factor de las emociones positivas y el aprendizaje, no se detecta correlación significativa para la confianza experimentada en la intervención. En base a estos resultados, sería recomendable que, a la hora de analizar las relaciones entre emociones y aprendizaje, no sólo se estudien los factores asociados a grupos de emociones para obtener una imagen global, sino también cada emoción a nivel individual.

Los resultados obtenidos concuerdan, además, con trabajos que han demostrado que la información relacionada con algún estímulo emocional se recuerda mejor que la información neutra (Dunsmoor et al. 2015). Por otro lado, las correlaciones observadas entre las emociones positivas (alegría, satisfacción, entusiasmo y diversión) y los resultados de aprendizaje se ajustan a investigaciones previas que han demostrado que una activación de las emociones positivas puede dar lugar a mejores resultados de aprendizaje (Fredrickson 1998; Kensinger y Corkin 2004), ya que estas provocan en la amígdala una excitación que mejora la memoria.

Las asociaciones detectadas entre emociones y aprendizaje no permiten establecer una relación causa efecto; pero sugieren que las emociones mejoran el aprendizaje, que el aprendizaje estimula a las emociones o que ambos fenómenos ocurren a la vez. Es decir, el aprendizaje podría haber sido provocado bien a través de la relación que tienen las actividades excitantes con la memoria (Kensinger y Corkin 2004) y la atención (Aydogan et al. 2015), o bien a través de la relación entre las emociones positivas y la motivación (Bradley 2000). En consecuencia, son precisas futuras investigaciones con las que profundizar en la influencia de las emociones en el aprendizaje, y con las que determinar, más allá de las interacciones detectadas entre emociones y aprendizaje, posibles relaciones de causalidad. Con todo, los resultados revelan que existe una asociación significativa entre ambas variables, puesto que las personas están motivadas para recordar detalles de eventos emocionales, dado que esta información es útil para predecir y controlar acontecimientos importantes del futuro (Dunsmoor et al. 2015). Así, esta asociación entre emociones y aprendizaje concuerda con el papel evolutivo de las emociones (Damasio 2010).

\section{$¿$ Tienen las emociones un valor predictivo en el aprendizaje? Análisis de las relaciones entre la expectativa de emociones ante una práctica de Biología y los resultados de aprendizaje}

En relación al análisis de las correlaciones entre la expectativa de emociones de los participantes, ante una práctica de Biología incluida en su formación como maestros, y los resultados de aprendizaje alcanzados tras esta; no se han detectado asociaciones significativas, a excepción del entusiasmo (Tabla 3). Se observa una correlación positiva entre la nota del postest y la intensidad esperada de entusiasmo ( $\mathrm{p}$-valor=.04). Este resultado indica que aquellos alumnos que esperaban experimentar más entusiasmo durante la intervención son aquellos que han obtenido mejores resultados de aprendizaje, sugiriendo que la intensidad previa de entusiasmo podría tener valor predictivo respecto al aprendizaje. Este resultado concuerda con estudios previos (Pekrun et al. 2002; Ruthig et al. 2008) que han mostrado que una alta intensidad de emociones positivas, y en concreto de entusiasmo, predice buenos resultados de aprendizaje. Estas investigaciones han hallado valor predictivo en el aprendizaje para altas intensidades previas de otras emociones positivas (en concreto orgullo e ilusión). En el presente estudio, sin embargo, no se ha detectado ninguna correlación significativa para las otras emociones positivas analizadas. Respecto a las emociones negativas, en estos y otros trabajos (Pekrun et al. 2010) se ha detectado que una alta intensidad de ellas (como el aburrimiento) predice malos resultados de aprendizaje. Sin embargo, en esta investigación no 
se han detectado asociaciones significativas entre la intensidad previa de ninguna emoción negativa y los resultados de aprendizaje. Por tanto, se requieren futuras investigaciones con los que ahondar en la importancia de las emociones previas de los estudiantes ante una actividad y su posible influencia en los resultados de aprendizaje a alcanzar.

Tabla 3. Coeficientes de correlación de la intensidad de las emociones previas a la intervención con los resultados de aprendizaje alcanzados tras esta (nota del postest). En negrita se resaltan las correlaciones significativas (Correlación de Spearman, ${ }^{*}$ p-valor $<.05$ )

\begin{tabular}{|c|c|}
\hline $\begin{array}{c}\text { Expectativa de emociones ante una } \\
\text { práctica activa de Biología }\end{array}$ & $\begin{array}{c}\text { Coeficiente de correlación con los resultados de } \\
\text { aprendizaje }\end{array}$ \\
\hline Alegría & .114 \\
\hline Confianza & -.089 \\
\hline Satisfacción & .067 \\
\hline Entusiasmo & .157 \\
\hline Diversión & .062 \\
\hline Nerviosismo & .074 \\
\hline Aburrimiento & -.078 \\
\hline Frustración & -.054 \\
\hline Preocupación & -.024 \\
\hline Incertidumbre & -.015 \\
\hline
\end{tabular}

\section{Implicaciones formativas}

En base a los resultados de la presente investigación, y teniendo en cuenta el papel de las emociones en la enseñanza-aprendizaje de las ciencias (Bradley 2000; Mega et al. 2014; Mellado et al. 2014), pueden establecerse algunas implicaciones educativas, sobre todo en lo referente a la formación inicial de maestros. De este modo, teniendo en cuenta las interacciones detectadas entre el nivel de conocimientos previos de Biología de ESO y algunas emociones negativas (aburrimiento, frustración y preocupación), sería conveniente abrir una reflexión acerca de la influencia del nivel de conocimientos previos científicos de ESO, de los maestros en formación inicial, en sus emociones negativas hacia las ciencias y su enseñanza. El diseño e implementación de sesiones basadas en estas interacciones podría ser relevante para contribuir a la disminución de las emociones negativas de los futuros maestros (Mellado et al. 2014, Damasio 2010), así como para contribuir a evitar la transferencia de estas en su futura acción docente (Frenzel et al. 2009). Por otro lado, la asociación positiva detectada entre la intensidad previa de entusiasmo y los resultados de aprendizaje pone de manifiesto la necesidad de considerar las emociones previas de los futuros maestros a la hora de implementar con estos actividades en el aula o el laboratorio, recomendando fomentar un clima emocionalmente positivo ante la implementación de dichas actividades. Además, sería conveniente abordar estas interacciones conocimientos previos-emociones y emociones previas-aprendizaje con los maestros en formación, en sesiones en las que se forme a estos en competencias emocionales (Mellado et al. 2014). Esto puede resultar relevante para que estos alumnos tengan en cuenta estas interacciones en su futuro desempeño profesional.

Respecto a las interacciones emociones-aprendizaje durante la práctica activa de Biología implementada, a pesar de no indicar una relación causa-efecto entre el dominio afectivo y el cognitivo, los resultados de esta investigación apoyan que una intervención educativa en la que se consideren las emociones de los alumnos podría mejorar los resultados de aprendizaje. Los coeficientes de correlación, entre las emociones y la nota del postest, muestran en qué medida una modificación en las emociones experimentadas por el alumnado, en las prácticas de Biología, podría mejorar los resultados de aprendizaje de esta disciplina. En concreto, sugieren que el aprendizaje de Biología, de maestros en formación inicial, podría ser favorecido si se plantearan actividades prácticas activas con las que se disminuyesen las emociones negativas, fundamentalmente preocupación y frustración, y se aumentasen las emociones positivas; 
especialmente alegría, satisfacción, entusiasmo y diversión. Por otro lado, estos resultados podrían tener interesantes implicaciones en el diseño de los programas formativos de los futuros maestros. Así, podría ser valioso incluir, en los procesos de formación inicial del profesorado, secuencias de enseñanza-aprendizaje con las que se adquieran estrategias para el desarrollo de este tipo de prácticas activas, motivantes para los alumnos de Educación Primaria. Esto es necesario ya que, como afirman Fernández-Abascal et al. (2001), los buenos profesores de ciencias deben sustituir las emociones negativas de sus alumnos por emociones positivas, a través del planteamiento de actividades científicas creativas y emocionantes. Además, la implementación de estas actividades en aulas de Educación Primaria con alumnado de este nivel educativo, por parte de maestros (tanto en ejercicio como en formación inicial durante sus prácticas de enseñanza), constituye una importante futura línea de investigación.

\section{Conclusiones}

Los resultados de la presente investigación secundan la existencia de una serie de interacciones entre algunas de las emociones experimentadas por una muestra de futuros maestros, antes y después de la implementación de una práctica activa de Biología, su nivel de conocimientos previos de Biología de Educación Secundaria y los resultados de aprendizaje adquiridos tras dicha actividad. Respecto a los objetivos planteados, los resultados revelan que:

- Bajos niveles de conocimientos previos de Biología del nivel de ESO están relacionados con altas intensidades previas de aburrimiento, frustración y preocupación ante una actividad práctica del presente.

- Las intensidades de alegría, entusiasmo, satisfacción y diversión experimentadas durante una práctica activa de Biología se asocian positivamente con los resultados de aprendizaje obtenidos con esta; así como que estos se asocian negativamente con las intensidades de frustración y preocupación.

- La intensidad de entusiasmo descrita antes de la implementación de la intervención se asocia positivamente con el aprendizaje obtenido tras esta, lo que podría indicar que la intensidad previa de esta emoción tiene valor predictivo con respecto a los resultados de aprendizaje.

\section{Agradecimientos}

Este estudio ha sido financiado por el Proyecto de Investigación EDU2016-77007-R del Ministerio de Economía y Competitividad y por la Ayuda a Grupos GR18004 de la Junta de Extremadura y el Fondo de Desarrollo Regional. El autor agradece al Ministerio de Educación, Cultura y Deportes la beca FPU de la que es beneficiario.

\section{Referencias}

Aydogan H., Bozkurt F., Coskun H. (2015) An Assessment of Brain Electrical Activities of Students toward Teacher's Specific Emotions. International Journal of Social, Behaviors, Educational, Economic, Business and industrial Engineering 9(6), 1977-2000

Banet E., Ayuso, G. (2003) Teaching of biological inheritance and evolution of living beings in secondary school. International Journal of Science Education 25(3), 373-407

Bisquerra R. (2000) Educación emocional y bienestar. Barcelona: Praxis

Bradley M. (2000) Emotion and motivation. Handbook of psychophysiology 2, 602-642

Brígido M., Borrachero A. B., Bermejo M. L., Mellado V. (2013) Prospective primary teachers' self-efficacy and emotions in science teaching. European Journal of Teacher Education 36(2), 200-217 
Caballero M. (2008) Algunas ideas del alumnado de secundaria sobre conceptos básicos de genética. Enseñanza de las ciencias 26(2), 227-244

Chattopadhyay A. (2005) Understanding of genetic information in higher secondary students in northeast India and the implications for genetics education. Cell Biology Education 4(1), 97-104

Damasio A. (2010) Y el cerebro creó al hombre. Barcelona: Destino

Dunsmoor J., Murty V., Davachi L., Phelps E. (2015) Emotional learning selectively and retroactively strengthens memories for related events. Nature 520, 345-348

Eldar E., Niv, Y. (2015) Interaction between emotional state and learning underlies mood instability. Nature communications 6, 6149-6159

Eldar E., Rutledge R., Dolan R., Niv Y. (2016) Mood as representation of momentum. Trends in Cognitive Sciences 20(1), 15-24

Fernández-Abascal E., Díaz M., Sánchez J. (2001) Procesos psicológicos. Madrid: Ediciones Pirámide

Foy P., Arora A., Stanco G. M. (2013) TIMSS 2011 User Guide for the International Database. Supplement 1: International Version of the TIMSS 2011 Background and Curriculum Questionnaires. Amsterdam: International Association for the Evaluation of Educational Achievement

Frenzel A., Goetz T., Lüdtke O., Pekrun R., Sutton R. (2009) Emotional transmission in the classroom: exploring the relationship between teacher and student enjoyment. Journal of educational psychology 101(3), 705-716

Fredrickson B. (1998) What good are positive emotions? Review of general psychology 2(3), 300319

García-Barros S., Martínez-Losada C. (2001) Qué actividades y qué procedimientos utiliza y valora el profesorado de educación primaria. Enseñanza de las Ciencias 19(3), 433-452

Gonzalez-Weil C., Harms U. (2012) Del árbol al cloroplasto: concepciones alternativas de estudiantes de $9^{\circ}$ y $10^{\circ}$ grado sobre los conceptos «ser vivo» y «célula». Enseñanza de las ciencias 30 (3), 31-52

Gormally C., Brickman, P., Hallar B., Armstrong N. (2009) Effects of inquiry-based learning on students' scince literacy skills and confidence. International journal for the scholarship of teaching and learning 3(2), 16-22

Gu X., Liu X., Van Dam N., Hof P., Fan J. (2013) Cognition-emotion integration in the anterior insular cortex. Cerebral Cortex 23(1), 20-27

Kensinger E., Corkin S. (2004) Two routes to emotional memory: Distinct neural processes for valence and arousal. Proceedings of the National Academy of Sciences of the United States of America 101(9), 3310-3315

Marcos-Merino J. M., Esteban R. (2018). Concepciones alternativas sobre Biología Celular y Microbiología de los maestros en formación: implicaciones de su presencia. Campo Abierto 6(2), 167-179

Marcos-Merino J. M., Esteban R., Gómez J. (2016). Efecto de una práctica docente diseñada partiendo de las emociones de maestros en formación bajo el enfoque Ciencia, Tecnología y Sociedad. Indagatio didactica 8(1), 143-157 
Marcos-Merino J. M., Esteban R., Gómez J. (2019). Formando a futuros maestros para abordar los microorganismos mediante actividades prácticas. Papel de las emociones y valoraciones de los estudiantes. Revista Eureka sobre Enseñanza y Divulgación de las Ciencias 16(1), 1602-1618

Mauss I., Robinson M. (2009) Measures of emotion: A review. Cognition and emotion 23(2), 209237

Mega C., Ronconi L., De Beni R. (2014) What makes a good student? How emotions, selfregulated learning, and motivation contribute to academic achievement. Journal of Educational Psychology 106(1), 121-131

Mellado V., Borrachero A., Dávila M., Melo L., Brígido M., Cañada F., Conde C., Costillo E., Cubero J., Esteban R., Martínez G., Ruiz C., Sánchez J., Garritz A., Mellado L., Vázquez B., Jiménez R., Bermejo M. (2014) Las emociones en la enseñanza de las ciencias. Enseñanza de las ciencias 32, 11-36

Mora F. (2008) El reloj de la sabiduría. Tiempos y espacios en el cerebro bumano. Madrid: Alianza Editorial

Pekrun R., Elliot A., Maier M. (2006) Achievement goals and discrete achievement emotions: A theoretical model and prospective test. Journal of Educational Psychology 98, 583-597

Pekrun R., Elliot A., Maier M. (2009) Achievement goals and achievement emotions: Testing a model of their joint relations with academic performance. Journal of Educational Psychology 101(1), 115-135

Pekrun R., Goetz T., Daniels L., Stupnisky R., Perry, R. (2010) Boredom in achievement settings: Exploring control-value antecedents and performance outcomes of a neglected emotion. Journal of Educational Psychology 102(3), 531-549

Pekrun R., Goetz T., Frenzel A., Barchfeld P., Perry R. (2011) Measuring emotions in students' learning and performance: The Achievement Emotions Questionnaire (AEQ). Contemporary educational psychology 36(1), 36-48

Pekrun R., Goetz T., Titz W., Perry R. (2002) Academic emotions in students' self-regulated learning and achievement: A program of qualitative and quantitative research. Educational psychologist 37(2), 91-105

Reigosa C., Jiménez M. (2000) La cultura científica en la resolución de problemas en el laboratorio. Enseñanza de las ciencias 18, 275-284

Retana D. A., De las Heras-Pérez M. Á., Vázquez-Bernal B., Jiménez-Pérez R. (2018) El cambio en las emociones de maestros en formación inicial hacia el clima de aula en una intervención basada en investigación escolar. Revista Eureka sobre Enseñanza y Divulgación de las Ciencias 15(2), 1-16

Ruthig J. C., Perry R., Hladkyj S., Hall N. C., Pekrun R., Chipperfield J. G. (2008) Perceived control and emotions: Interactive effects on performance in achievement settings. Social Psychology of Education 11(2), 161-180

Smith M., Wood W., Knight J. (2008) The genetics concept assessment: a new concept inventory for gauging student understanding of genetics. CBE-Life sciences Education 7(4), 422-430 


\section{Anexo 1}

Responde a las siguientes preguntas (sólo una opción es correcta)
1. Podemos extraer material genético de:
a) Sólo los seres vivos
b) Sólo los procariotas
c) Sólo los eucariotas
d) De todos los seres vivos y algunos no vivos (por ejemplo virus)

\section{2. ¿Dónde crees que se encuentra el ADN en un tomate?:}

a) Sólo en las células de la semilla

b) Sólo en las células de la pulpa (del fruto)

c) Sólo en las células de la piel

d) En todas las células

\section{El ADN es:}

a) Un ácido nucleico

b) Una grasa rodeada de fósforo

c) Una proteína

d) Un aminoácido

4. En los humanos los cromosomas sexuales se encuentran exclusivamente en:

a) Las células de los testículos y los ovarios

b) Todas las células con núcleo

c) Los espermatozoides y los óvulos

d) Las mitocondrias de todas las células

5. Qué tipos de células tienen mitocondrias y cloroplastos:

a) Las células animales

b) Las células vegetales

c) Las bacterias

d) Ninguna, o bien tiene mitocondrias o bien tienen cloroplastos

6. La diferencia esencial entre célula procariota y célula eucariota radica en:

a) El tamaño celular

b) La pared celular

c) El núcleo celular

d) La composición química del citoplasma

\section{Respecto al ADN señala la respuesta CORRECTA:}

a) Se encuentra exclusivamente en el núcleo celular

b) Es el único ácido nucleico de la célula

c) Se encuentra en cloroplastos, mitocondrias y núcleo

d) Está formado por aminoácidos

\section{Señala la afirmación CORRECTA:}

a) Todas las células están rodeadas por una membrana plasmática rígida

b) La membrana plasmática está formada fundamentalmente por lípidos, proteínas, carbohidratos y 
nucleótidos

c) Dentro de las células algunos orgánulos están rodeados por membranas similares a la membrana plasmática

d) Solo las células eucariotas tienen membrana plasmática

\section{En los vegetales, la pared celular es:}

a) Una estructura extracelular formada por polisacáridos, fundamentalmente celulosa

b) Una estructura rígida que las rodeas

c) La materia prima para formar el papel

d) Todas las respuestas son correctas

10. Identifica la relación "tipo celular - características" INCORRECTA:

a) Todas las células vegetales tienen pared celular

b) Todas las células animales tienen mitocondrias y cloroplastos

c) Todas las células vegetales tienen mitocondrias y cloroplastos

d) Todas las células animales tienen mitocondrias

11. Los riñones son órganos que se encuentran en el cuerpo humano. A un hombre le sacaron uno de sus dos riñones cuando era joven porque estaba enfermo. Ahora tiene un hijo. ¿Cuántos riñones tuvo su hijo al nacer?

a) 1 , ya que solo se puede heredar lo que se tiene

b) 2, ya que no se han alterado sus genes

c) 2, ya que los riñones se regeneran

d) 1 o 2 según si hereda 1 o ninguno del padre y el de la madre.

12. ¿Cuál de las siguientes es la mejor descripción del propósito de la respiración celular?

a) Proporcionar energía para la actividad celular

b) Producir azúcar para almacenar en las células

c) Liberar oxígeno para la respiración

d) Proporcionar dióxido de carbono para la fotosíntesis

13. ¿Qué tipo de células destruyen a las bacterias que invaden el cuerpo?

a) Los glóbulos blancos

b) Los glóbulos rojos

c) Las células del riñón

d) Las células del pulmón

14. La imagen muestra una célula. ¿Cuál es la función de la parte de la célula marcada con una $X$ ?

a) Almacenar agua

b) Producir alimento

c) Absorber energía

d) Controlar las actividades

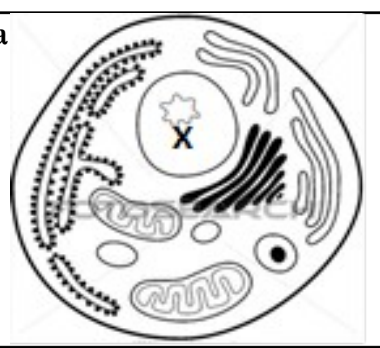

